\title{
The Complexity Based Analysis of the Correlation between Spider's Brain Signal and Web
}

\author{
Hamid R Namazi* \\ 167 Division Ave Brooklyn, New York 11211, USA
}

\author{
*Corresponding Author: Hamid R Namazi, 1167 Division Ave Brooklyn, New York 11211, USA, Email: \\ hrnneuromap@gmail.com
}

\begin{abstract}
Analyzing the brain activity in human and animals is the main research topic in neuroscience. In this way, the analysis of spider's brain has aroused the attention of some scientists. On the other hand, building fractal web as an important action of spiders(which mainly done for catching their food) has been studied widely. However, no one has discovered any relation between the structure of spider's brain signal (as the feature of brain activity) and the structure of its web. This study reveals the relation between the complexity of spider's web and its brain signal complexity. We demonstrated that fractal structure of spider's web shifts towards the fractal structure of its brain signal. In another step, we also analyzed the correlation between spider's web entropy and its brain signal entropy. The results of analysis showed for the first time that spider with more fractal brain signal builds the web that is more fractal. The similar result has been obtained in case of entropy analysis of spider's web and brain signal. The capability observed in this research opens a new door to scientists to study the relation between different animals' behaviors (such as walking) and their brain activity.
\end{abstract}

Keywords: Spider, Brain signal, Spider's web, Complexity, Fractal structure.

\section{INTRODUCTION}

Between all studies done on animal behavior, spiders have aroused the attention of some scientists[1-5].An important behavior of spiders is building the web and several works have been done on studying this behavior [6-7].Between these works, few scientists have tried to analyze the behavior of spider on building its web by applying drugs on spider. The first study refers to the work done by Witt [8] in 1948. He tested garden spiders with a range of psychoactive drugs and found out that drugs affect the size and shape of the web rather than the time when it is built. Based on the results, the shape of the spider's web changed more, and its design became irregular at higher doses of drug application. In another work which was done by a NASA research group, Noever et al. [9] repeated Witt's investigation on the influence of psychoactive drugs on European garden spiders. They did their investigation in order to study the effect of toxicity of drugs on spiders. They digitized and processed the images of the web cells in order to compute numbers of cells and average areas, perimeters, and radii of cells. They found out that a more toxic drug causes a more deformed web (more slides the spider fails to complete)in comparison with a normal web. Also please look at [10-11].

Besides all efforts done on studying the spider's behavior on building its web, no work has been reported yet which relates the spider's web to its brain activity. Now, the question which arises here is that how spider's brain activity affects its web. Here, in order to make a link between these two features, we benefit from the concept of fractal.

Fractal theory [12] can be used to study the scaling properties of the spider's web. A fractal is a natural phenomenon or a mathematical set that exhibits a repeating pattern that displays at every scale (self-similar). The scaling rules are characterized by "scaling exponents" (dimension). "Simple" regular fractals have integer scaling dimensions. Complex selfsimilar objects have non-integer dimension. Fractals can be defined as geometric objects whose scaling exponent (dimension) satisfies the Szpilrajn inequality [13]:

$$
\aleph \geq D_{T}
$$

Where אis the scaling exponent (dimension) of the object and $D_{T}$ is its topological dimension, 
that is, Euclidean dimension of units from which the fractal object is built. In fact, Fractal and Euclidean geometries are conjugate approaches. Fractal geometry builds complex objects by applying simple processes to complex building blocks; Euclidean geometry uses simpler building blocks but frequently requires complex building processes [13].Fractal dimension can be viewed as an index of complexity, which shows how a detail in a pattern changes with the scale at which it is measured. Fractal approach has been used widely in order to study the complexity of different processes [14-22].

In this research, for the first time we analyze the relation between spider's brain activity and its web. As spider's brain signal and web are complex, and the activity of spider is organized by its brain, we benefit from the concept of complexity and hypothesize that the complexity of spider's brain signal should affect its web complexity. To test this hypothesis, for the first time we investigate the correlation between the fractal nature of the spider's web and the fractal structure of its brain signal in different conditions. In order to explain the results of works done in [8] and [9], we chose drugs for changing spider's brain activity. As another novelty, we also chose entropy in order to make a link between the spider's web and its brain signal.

\section{Method}

We aim to investigate the correlation between the fractal structure of spider's web and the fractal dynamics of its brain signal. In order to stimulate the spider's brain, three different drugs(caffeine, Benzedrine and chloral hydrate) are given to the spider in separate experiments, and accordingly the structure of its brain signal and the built web are analyzed by computing fractal dimension. In this way, we link the complexity of spider's web to the complexity of its brain signal.

As was mentioned before, fractal dimension stands for the complexity of a structure [23]. The fractal dimension can be determined using techniques that approximate scaling and detail from limits estimated from regression lines of $\log -\log$ plot of size versus scale. There are several formal mathematical definitions such as box counting, information dimension and correlation dimension for computation of fractal dimension [24]. All these methods can be seen as special cases of a continuous spectrum of generalized fractal dimensions. Here we define this generalized fractal dimension. The fractal exponent is based on the entropy concept for a probability distribution [25]. For instance, in case of a time series with range of variation between $V_{\text {max }}$ and $V_{\text {min }}$, we can divide the total range into $\mathrm{N}$ bins, where each bin has the size of $\delta \in$ :

$\mathrm{N}=\frac{\mathrm{V}_{\max }-\mathrm{V}_{\min }}{\delta \in}$

So, the probability of a value to fall into the i'th bin:

$$
\mathrm{w}_{\mathrm{i}}=\lim _{\mathrm{N} \rightarrow \infty} \frac{\mathrm{N}_{\mathrm{i}}}{\mathrm{N}}
$$

In Equation (3) $N_{i}$ is the number of times the value falls into the i-th bin. For a time series, it can be written as:

$\mathrm{w}_{\mathrm{i}}=\lim _{\mathrm{T} \rightarrow \infty} \frac{\mathrm{t}_{\mathrm{i}}}{\mathrm{T}}$

where $t_{i}$ stands for the total time that the value spends in the $\mathrm{i}$-th bin, and $\mathrm{T}$ is total time.

The generalized fractal dimension of order $\mathrm{q}$ is defined by [26]:

$\mathrm{D}_{\mathrm{q}}=\lim _{\in \rightarrow 0} \frac{1}{\mathrm{q}-1} \frac{\log \sum_{\mathrm{i}=1}^{\mathrm{N}} \mathrm{w}_{\mathrm{i}}^{\mathrm{q}}}{\log \epsilon}$

where $\in$ is the scaling factor.

In the second step, we employ Shannon entropy in order to study the correlation between the information contents of spider's brain signal and its web. Shannon entropy stands for the information content of the process (signal or image) [27]. In fact, the signal or image which has a bigger value of Shannon entropy contains more information. In general, Shannon entropy is defined as:

$H=-\sum_{i} p_{i} \log p_{i}$

where $p_{i}$ is the probability of character number $i$ showing up in a stream of characters of the given "script". In case of an image, $p_{i}$ is the probability of occurrence for each symbol / intensity that is encountered in the image. Therefore, this probability can be defined by finding the total number of pixels observed for every possible intensity in the image and normalizing it by the total number of pixels.

\section{Data Collection}

The experiments were conducted on 20 female European garden spiders. We did not do experiments on male spiders because male spiders eat less, and do not build webs as often. The spiders were given a constant source of moisture and sustained on a diet of domestic 
crickets. In fact as we are interested that spiders build web, based on literature [8]we sustain the spiders on the diet to encourage them to build a new web each night to hunt for their food.

To ensure that changes in the brain signals were primarily due to the applied drugs, the spiders were shielded from external stimuli. Experiments were performed in an electrically shielded, acoustically isolated, and dimly illuminated room.

In order to stimulate the spider's brain, we applied three drugs on spiders. These drugs were same as drugs used in the research done by the NASA research group [9], which consists of caffeine, Benzedrine, and chloral hydrate.

In order to feed spiders with the drug, based on Witt's method [8] we mixed $10 \mu g$ of the drug with sugar water to attract interest, and administered tiny drops of the solutions into the spiders' mouths (one drug per spider) using a $0.5 \times 16 \mathrm{~mm}$ sterile syringe.

As spiders typically build their webs between 2AM to 5AM and drug application doesn't change the spiders' tendency to work on webs late at night, the drug was applied on spiders at $10 \mathrm{pm}$, and we waited for the spider to start building its web. In order to collect the spider's web, we designed a transparent Perspex frame with the size of $50 \mathrm{~cm} \times 50 \mathrm{~cm}$ which corresponds to the size of European garden spider's web that is up to $40 \mathrm{~cm}$ in diameter. We put the spider in the frame and closed the frame using cling wrap to prevent spider from escaping.

After the spider has finished its web, we removed the spider from the web without distorting its web. We photographed the web before and after application of drugs using 18 MP Cannon DSLR camera with $55 \mathrm{~mm}$ lens. Before photography, we made the frame's background very dark and applied bright light from sides of the frame using $15 \mathrm{~W}$ fluorescent bulbs.

In this research, we recorded the spider's brain signal in two steps. One recording was done before the application of the drug to the spider. The recording provides the amplitude variations of the brain signal for 60 seconds. After we photographed the spider's web, we repeated the recording. In this step also, we recorded 60 seconds of the spider's brain signal. For brain signal collection, we benefit from the method developed by Menda et al [28].
For this purpose, we designed a non-invasive plastic platform that holds the spider and prevents its movement during recordings. In addition, in order to limit spider's mobility we cold anesthetized spiders at $-5^{\circ} \mathrm{C}$ for about 3 minutes, and then waxed them in place by Kerr dental sticky wax using a cool soldering iron.

An extracellular glass-insulated tungsten microelectrode was inserted through the small hole in the prosoma (rightward of the back midline) and then advanced into the brain in or just posterior to the arcuate body. A AmScope SM-4TZ-144-5MT Trinocular Stereo Microscope was used to identify the location of the electrode entry point, based on external morphological features on the prosoma (akin to the cephalothorax) of the spider. The hole which was made using the tip of a sharp $0.5 \times 16 \mathrm{~mm}$ sterile syringe had small size (approximately $120-180 \mathrm{~mm}$ in diameter) to prevent the catastrophic fluid loss.

The recording electrode was coated in fluorescent dye before the experiment begins, in order to verify the locations of recording site in the central system. This job allows the subsequent visualization of the recording site.

Pre-insertion orientation of the microelectrode was executed using a stereotaxic micro manipulator. A hydraulic micro drive with resolution up to $1 \mu \mathrm{m}$ was used to advance the electrode. A second sharp tungsten electrode (as a ground) was inserted into the opisthosoma. Resultant electrical activity from the recording electrode was collected using a head stage and its software. The signal was digitized using an analog-to-digital converter.

Data collection in case of each drug has been done in different days. On the first day, we collected the spider's brain signal and web pattern without any drug. On the second day, we tested the spider's behavior for Caffeine. This procedure continued to test Benzedrine and Chloral hydrate in the following days. At the conclusion of all experiments, the spiders were euthanized by placing them in a freezer at approximately $-6^{\circ}$. It should be mentioned that one pharmacologist and one zoologist monitored all steps of experiments and data collection.

\section{Data Analysis}

Since the collected brain signal was noisy, first it was band-pass filtered. Then, fractal dimension and Shannon entropy of the filtered 
signal were computed. The computation of fractal dimension was based on the box counting method [24]. In the similar way, the computations of fractal dimension and Shannon entropy for the spider's web have been done.

All of the analyses were done in MATLAB. It is noteworthy as the collected photos showed the web's color in white and the background color in black, before computation of fractal dimension and Shannon entropy we changed the web's color to black and the background color to white using Adobe Photoshop CC (16.0).

\section{Statistical ANAlysiS}

Mean values for the dependent variables (fractal dimension and Shannon entropy of spider's web) were compared across no drug and drug feeding conditions with one-way repeated measures ANOVA [29]. Mauchly's test with the significance level $(\alpha)$ of 0.05 was conducted in order to test for sphericity. As it is known Mauchly's sphericity test is used to validate ANOVA. In order to analyze the trend between the variations of the spider's web versus its brain signal, we did trend analysis. For a repeated measures design, we used Omega squared $\left(\omega^{2}\right)$ [30] as an unbiased measure of effect size suitable for small samples; In case of pair wise comparisons we used effect size, $r$. Pearson's correlation coefficient $(r)$ is used in order to quantify some type of correlation and dependence between two or more random variables or observed data values.

\section{RESUlT}

Here we report the result of analysis, which investigates the correlation between the brain activity and the web of European garden spiders. It should be noted that considering 40,000 described spider species [31], we cannot give specific information for each species.
In general, the application of drugs caused the spider to become very drowsy and spins its web with huge gaps. Figure 1 shows the samples of spider's web (collected in our experiments) in different conditions. As it is clear in this figure, web patterns changed enormously in case of each drug application.
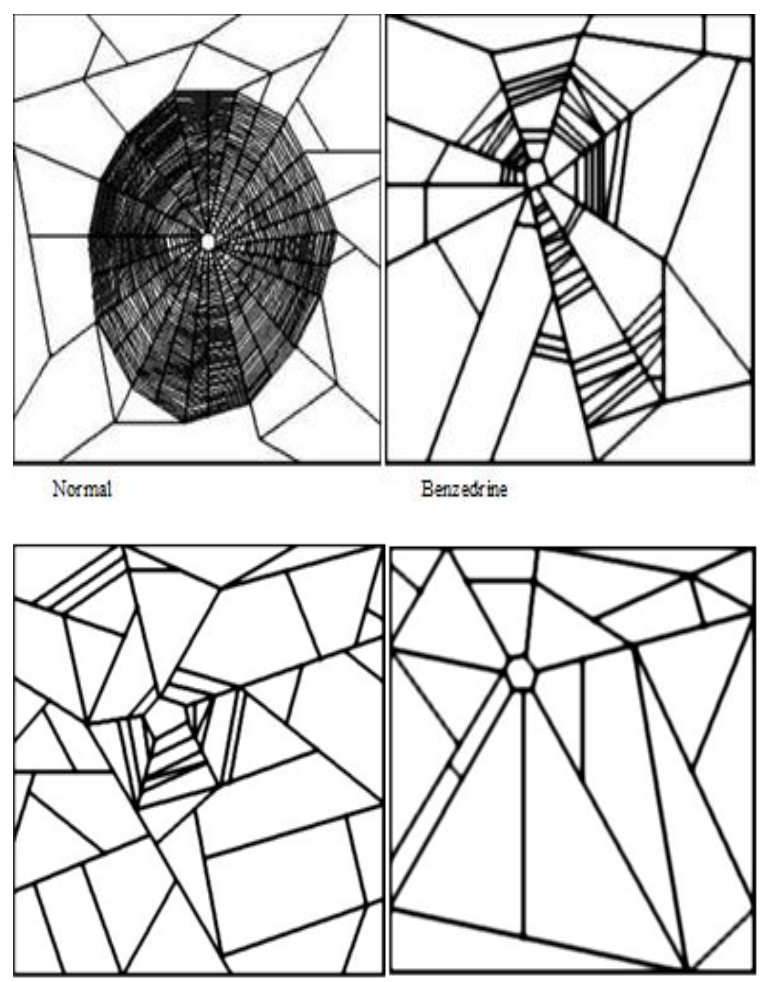

Caffeine

Chloral hydrate

Figure1. Samples of spider's web in different conditions

Here we report the result of analysis. It is noteworthy that all trials were included in the analysis. Figure 2 shows the variations of web's fractal dimension versus the variations of fractal dimension for the spider's brain signal in case of different drugs. The result in case of each drug indicates the averaged value between all spiders.

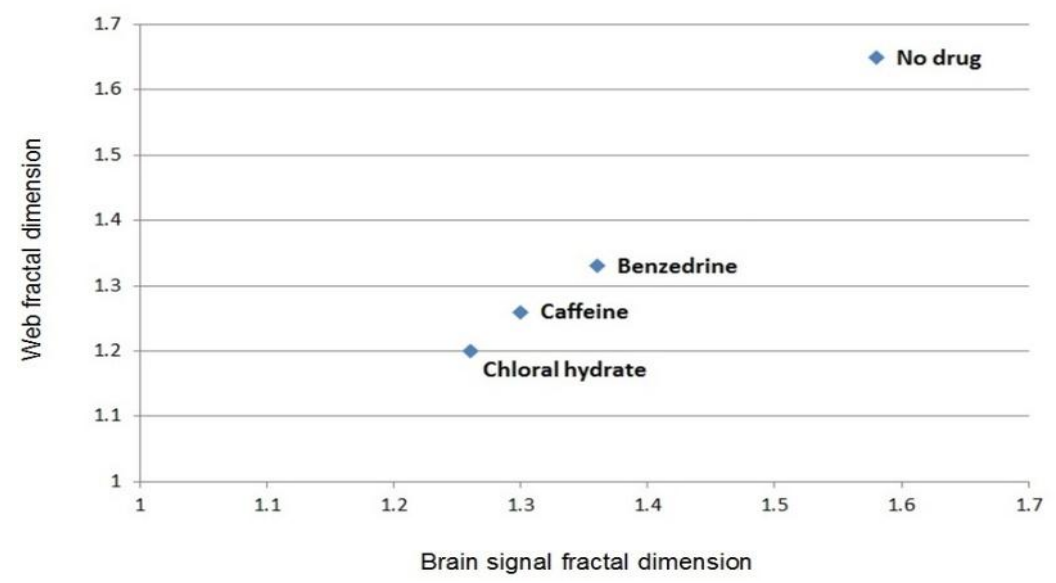

Figure2. Scatter plot showing the spider's web fractal dimension versus the spider's brain signal fractal dimension in case of different drugs. 
Considering $\quad F_{\text {crit }}(3,76)=2.72$ at $\quad \alpha=0.05$, the result of statistical analysis $[F(3,76)=402$, $\mathrm{p}=0.0001]$ indicates the significant influence[33] of drugs on the fractal structure of the spider' web, with an effect size $\omega^{2}=0.94$. In general, the application of drugs decreased the fractal dimension of the brain signal and the built web. As fractal dimension stands for complexity, it can be said that the application of drug decreased the complexity of the spider's brain signal and accordingly its web. A significant linear trend between the complexity of spider's brain signal in different drug feeding conditions was observed, that as it is evident in Figure 2, by moving from "no drug" condition to "Chloral hydrate", spider's brain activity yields a bigger decrement in the fractal dimension of the spider's web that mirrors the trend in reduction of fractal dynamics of spider's brain signal in case of these drugs. The effect sizes which are brought in Table 1 suggest that the lowest brain signal fractality in case of Chloral hydrate led to the greatest change in the fractal exponent of the spider's web observed across all Chloral hydrate comparisons. Based on this result, there is a correlation between the complexity of spider's brain signal and the complexity of its web.

Table1. Pair wise comparisons in different conditions

\begin{tabular}{|l|l|l|}
\hline Condition & $\begin{array}{l}\text { Fractal } \\
\text { dimension } \\
\text { Effect size (r) }\end{array}$ & $\begin{array}{l}\text { Shannon } \\
\text { entropy } \\
\text { Effect size (r) }\end{array}$ \\
\hline $\begin{array}{l}\text { No drug vs. } \\
\text { Benzedrine }\end{array}$ & 0.96 & 0.96 \\
\hline $\begin{array}{l}\text { No drug vs. } \\
\text { Caffeine }\end{array}$ & 0.98 & 0.98 \\
\hline $\begin{array}{l}\text { No drug vs. } \\
\text { Chloral } \\
\text { hydrate }\end{array}$ & 0.99 & 0.99 \\
\hline $\begin{array}{l}\text { Benzedrine } \\
\text { vs. Caffeine }\end{array}$ & 0.61 & 0.85 \\
\hline $\begin{array}{l}\text { Benzedrine } \\
\text { vs. Chloral } \\
\text { hydrate }\end{array}$ & 0.82 & 0.95 \\
\hline $\begin{array}{l}\text { Caffeine vs. } \\
\text { Chloral } \\
\text { hydrate }\end{array}$ & 0.60 & 0.84 \\
\hline
\end{tabular}

Figure 3 shows the variations of web's Shannon entropy versus the variations of Shannon entropy of spider's brain signal in case of different drugs. The result in case of each drug indicates the averaged value between all spiders.

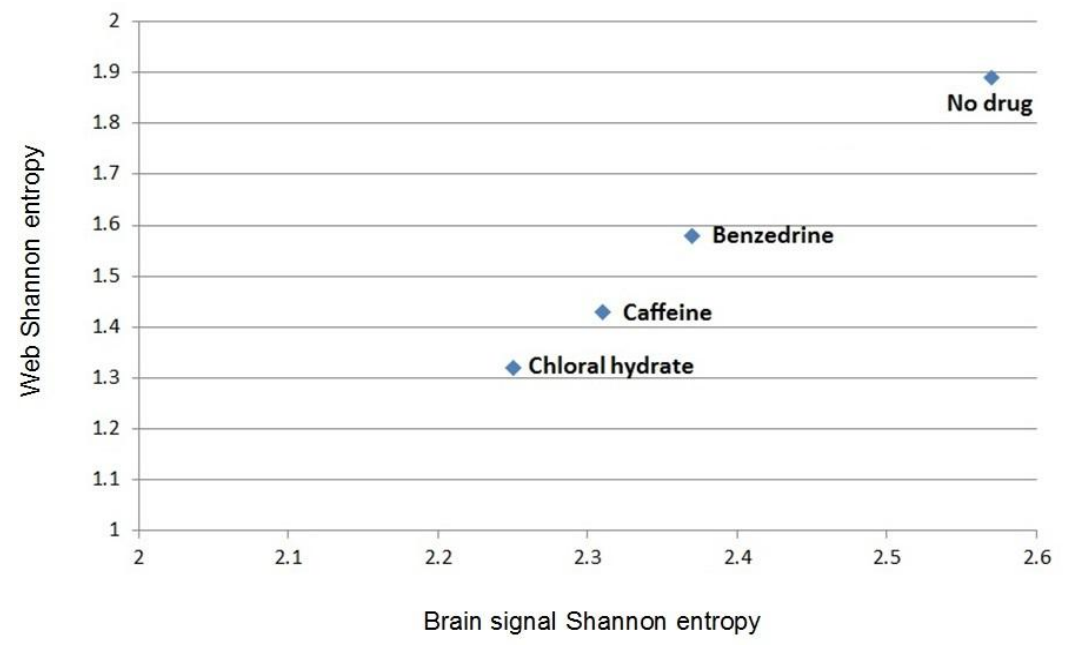

Figure3. Scatter plot showing the spider's web Shannon entropy versus the spider's brain signal Shannon entropy in case of different drugs

Considering $\quad F_{\text {crit }}(3,76)=2.72$ at $\quad \alpha=0.05$, the result of statistical analysis $[F(3,76)=$ $1225, \mathrm{p}=0.0001]$ indicates the significant influence of drugs on the entropy of the spider' web, with an effect size $\omega^{2}=0.96$. In general, the application of drugs decreased the Shannon entropy of the brain signal and the built web. As Shannon entropy stands for the information content, it can be said that the application of drug reduced the information content of the spider's brain signal and accordingly its web. A significant linear trend between the entropy of spider's brain signal in different drug feeding conditions was observed, that as it is evident in Figure 3, by moving from "no drug" condition to "Chloral hydrate", spider's brain activity yields a bigger decrement in the Shannon entropy of the spider's web that mirrors the trend in reduction of the Shannon entropy of the spider's brain signal in case of these drugs. The effect sizes that are brought in Table 1 suggest that the lowest brain signal entropy in case of Chloral hydrate led to the greatest change in the entropy of the spider's web observed across all Chloral hydrate comparisons. Based on this result, there is a correlation between the 
information content of the spider's brain signal and the information content of its web.

In summary, there is a correlation between the complexities of spider's web and brain signal, where the greater variation in the complexity and entropy of spider's brain signal causes the greater variation in the complexity and entropy of spider's web.

\section{CONCLUSION AND DISCUSSION}

For the first time we analyzed the correlation between the fractal dynamics of spider's brain signal and the fractal structure of its web. Our results demonstrated the relation between the complexities of the spider's web and brain signal, as the trend across the decrement of fractal exponent of the brain signal (due to drugs) is mirrored on the trend across the decrement of fractal exponent of the spider's web. For instance, Chloral hydrate had the greatest influence on the spider's brain signal fractal dimension. This greatest effect will accordingly cause the greatest influence on fractal dimension of spider's web compared to other drugs. As fractal dimension stands for complexity, it can be said that when the spider's brain signal is less complex, spider builds its web that is less complex. This behavior was seen in comparison between other conditions as well. In fact, the results of our analysis explain the results of works reported in [8] and [9].In explaining the founding in [9] which says the more toxic the chemical, the more deformed a web, we can say that when a drug is more toxic, it will have bigger influence on the spider's brain, which decreases the brain signal fractal dimension. Thus, accordingly spider will build its web that is less complex, having smaller value of fractal dimension. On the other hand, the result of our analysis showed the similar trend between the spider's brain signal entropy and its web entropy, where having smaller brain signal's entropy will result in web with the smaller entropy.

In overall, it can be said that on one side there is a correlation between the spider's brain signal fractal dimension and its web fractal dimension, and on the other side there is a correlation between the spider's brain signal entropy and its web entropy. As fractal dimension and entropy stand for the complexity and information content respectively, we can claim that the variation of the complexity and information content of the spider's brain signal is mapped on the variation of complexity and information content of its web.
Although the main purpose of this research was to study the relation between the spider's brain activity and its web, but based on the mentioned previous work on the analysis of the influence of drugs on spiders, where Witt [8] stated that spiders behaviors (such as drowsiness and walking) on psychoactive drugs are like humans on drugs, our further studies on the relation between the spider' brain activity and its web on psychoactive drugs may explain the relation between human brain activity and his behavior in case of psychoactive drug application, where doing these experiments on human is restricted by law and also doing the same experiments for higher animals is expensive and time consuming. Also, since spiders had different responses to different drugs, they could serve as an easy test for identifying unknown drug poisonings in humans.

\section{REFERENCES}

[1] Pfeiffer, K., Panek, I., Höger, U., French, A. S. \&Torkkeli, P. H. Random stimulation of spider mechanosensory neurons reveals long-lasting excitation by GABA and muscimol. J. Neurophysiol. 101, 54-66; DOI: 10.1152/jn.91020.2008 (2009).

[2] Schaber, C. F. \& Barth, F. G. Spider joint hair sensilla: adaptation to proprioreceptive stimulation. J. Comp. Physiol. A. Neuroethol. Sens. Neural. Behav. Physiol. 201, 235-48; DOI: 10.1007/s00359-014-0965-4 (2015).

[3] Gingl, E. \& French, A. S. Active Signal Conduction through the Sensory Dendrite of a Spider Mechanoreceptor Neuron. J. Neurosci. 23, 6096-6101 (2003).

[4] Trichilo, P. J. \& Wilson L. T. An ecosystem analysis of spider mite outbreaks: physiological stimulation or natural enemy suppression. Exp. Appl. Acarol. 17, 291-314 (1993).

[5] Blamires, S. J., Piorkowski, D., Chuang, A., Tseng, Y. H., Toft, S. \& Tso, I. M. Can differential nutrient extraction explain property variations in a predatory trap? R. Soc. Open Sci. 2: 140479; DOI: 10.1098/rsos. 140479 (2015).

[6] Coddington, J. A. \& Levi. H. W. Systematics and evolution of spiders (Araneae). Annu. Rev. Ecol. Evol. Syst. 22, 565-592 (1991).

[7] Benjamin, S. P. \&Zschokke, S. Homology, behaviour and spider webs: web construction behaviour of Linyphiahortensis and $\mathrm{L}$. triangularis (Araneae: Linyphiidae) and its evolutionary significance. J. Evol. Biol. 17, 120-130 (2004).

[8] Rainer, F. F. Biology of spiders (Oxford University Press, 2010).

[9] Noever, D. A., Cronise, R. J. \&Relwani, R. A. Using Spider-Web Patterns To Determine 
Toxicity. NASA Tech Briefs (ISSN 0145319X), 19 (4), 82 (1995).

[10] Witt, P. N. Drugs alter web-building of spiders: a review and evaluation. Behav. Sci. 16, 98-113 (1971).

[11] Foelix, R. Biology of spiders (Oxford University Press, 2011).

[12] Parke, G. A. R. \& Disney, P. Space Structures 5 (Thomas Telford, 2002).

[13] Kulish, V.V. Partial Differential Equations (Pearson, 2010).

[14] Namazi, H., Akrami, A. \& Kulish, V. V. The Analysis of the Influence of Odorant's Complexity on Fractal Dynamics of Human Respiration. Sci. Rep. 6, 26948; DOI: 10.1038/srep26948 (2016).

[15] Namazi, H., Kulish, V. V. \&Akrami, A. The analysis of the influence of fractal structure of stimuli on fractal dynamics in fixational eye movements and EEG signal. Sci. Rep. 6, 26639; DOI: 10.1038/srep26639 (2016).

[16] Anandarup, M., Nidhi, P. \&Anirban, R. Heart murmur detection using fractal analysis of phonocardiograph signals. Int. J. Comput. Appl. 88; DOI: 10.5120/15407-3928 (2014).

[17] Namazi, H. and Kulish, V. V. Fractal Based Analysis of the Influence of Odorants on Heart Activity. Sci. Rep. 6, 38555; doi: 10.1038/srep38555 (2016).

[18] Kyung-Hoe, H. et al. Fractal analysis of mandibular trabecular bone: optimal tile sizes for the tile counting method. Imaging Sci. Dent. 41, 71-78 (2011).

[19] Namazi, H., Kulish, V. V. \& Wong, A. Mathematical Modelling and Prediction of the Effect of Chemotherapy on Cancer Cells. Sci. Rep. 5, 13583; DOI: 10.1038/srep13583 (2015).

[20] Namazi, H. \& Kulish, V. V. Fractional Diffusion Based Modelling and Prediction of Human Brain Response to External Stimuli.
Comput. Math. Methods Med. 2015, 148534; DOI:10.1155/2015/148534 (2015).

[21] Namazi, H. \&Kiminezhadmalaie, M. Diagnosis of Lung Cancer by Fractal Analysis of Damaged DNA. Comput. Math. Methods Med. 2015; 1-13 (2015).

[22] Namazi, H., Akrami, A., Hussaini, J., Silva, O.N., Wong, A. \& Kulish, V.V. The fractal based analysis of human face and DNA variations during aging. Bioscience Trends. 2016; DOI: 10.5582/bst.2016.01182.

[23] Mandelbrot, B. B. The fractal geometry of nature (Macmillan, 1983).

[24] Annadhason, A. Methods of Fractal Dimension Computation. IRACST - International Journal of Computer Science and Information Technology \& Security (IJCSITS). 2, 166-169 (2012).

[25] Renyi, A. On a new axiomatic theory of probability. Acta Mathematica Hungarica. 6, 145-174 (1906).

[26] Schroeder, M. R. Fractals, Chaos, Power Laws: Minutes from an Infinite Paradise (W. H. Freeman and Company, 1991).

[27] Machta J. Entropy, information, and computation. Am. J. Phys. 67, 1074-1077 (1999).

[28] Menda, G., Shamble, P. S., Nitzany, E. I., Golden, J. R. \& Hoy, R. R. Visual perception in the brain of a jumping spider. Curr. Biol. 24, 2580-5; $\quad$ DOI: $\quad 10.1016 /$ j.cub.2014.09.029 (2014).

[29] Howell, D. Statistical Methods for Psychology (Wadsworth Gengage learning, 2010).

[30] Olejnik, S., Algina, J. Generalized Eta and Omega Squared Statistics: Measures of Effect Size for Some Common Research Designs. Psychol. Methods. 8, 434-447 (2003).

[31] Platnick, N. I. The world spider catalog. American Museum of Natural History (https://research.amnh.org/iz/spiders/catalog/).

Citation: Hamid R Namazi. The Complexity Based Analysis of the Correlation between Spider's Brain Signal and Web. ARC Journal of Neuroscience. 2017; 2(4):38-44. doi:dx.doi.org/10.20431/2456057X.0203006.

Copyright: () 2017 Authors. This is an open-access article distributed under the terms of the Creative Commons Attribution License, which permits unrestricted use, distribution, and reproduction in any medium, provided the original author and source are credited. 